\title{
Close-packed arrays of transition-edge x-ray microcalorimeters with high spectral resolution at $5.9 \mathrm{keV}$
}

\author{
N. Iyomoto, S.R. Bandler, R.P. Brekosky, A.-D. Brown, J.A. Chervenak, F.M. \\ Finkbeiner, R.L. Kelley, C.A. Kilbourne, F.S. Porter, J.E. Sadleir, and S.J. Smith \\ NASA/Goddard Space Flight Center, Greenbelt, MD 20771* \\ E. Figueroa-Feliciano \\ Department of Physics, Massachusetts Institute of Technology, Cambridge, MA 02139
}

(Dated:)

\begin{abstract}
We present measurements of high fill-factor arrays of superconducting transition-edge x-ray microcalorimeters designed to provide rapid thermalization of the x-ray energy. We designed an $\mathrm{x}$-ray absorber that is cantilevered over the sensitive part of the thermometer itself, making contact only at normal metal-features. With absorbers made of electroplated gold, we have demonstrated an energy resolution between 2.4 and $3.1 \mathrm{eV}$ at $5.9 \mathrm{keV}$ on 13 separate pixels. We have determined the thermal and electrical parameters of the devices throughout the superconducting transition, and, using these parameters, have modeled all aspects of the detector performance.
\end{abstract}

PACS numbers:

The requirements of the X-ray Microcalorimeter Spectrometer on NASA's Constellation-X mission include a full-width-at-half-maximum (FWHM) energy resolution $\Delta E$ of $2.5 \mathrm{eV}$ at count rates up to $1000 \mathrm{~s}^{-1}$ per pixel and at least $95 \%$ quantum efficiency (QE) at an x-ray energy $E$ of $6 \mathrm{keV}$. In order to cover a 5-arcminute field of view with spatial sampling matched to the performance of the optics, a $64 \times 64$ array of 0.25 -mm pixels with a high fill factor is required. We report significant progress in the development of superconducting transition-edge sensor (TES) microcalorimeter ${ }^{1}$ arrays for missions such as Constellation-X.

The TES thermometers in our arrays consist of bilayers of $\mathrm{Mo}$ and $\mathrm{Au}$ with a superconducting transition temperature $T_{\mathrm{c}}$ of $\sim 0.1 \mathrm{~K}$. Each 0.13 -mm square TES is centered on a $0.5-\mu \mathrm{m}$ silicon-nitride membrane, which is the thermal link with thermal conductance $G$ to the solid silicon frame at $T_{\mathrm{b}} \sim 50 \mathrm{mK}$. The TES pixels are arrayed on a $0.25-\mathrm{mm}$ pitch. To achieve high $\mathrm{QE}$ and fill factor requires thick-film $\mathrm{x}$-ray absorbers that fill the space above and between each TES. Our original approach to this requirement was to construct $\mathrm{Bi} / \mathrm{Cu}$ absorbers that make contact to the entire area of the TES, but extend cantilevered over the surrounding area. However, we determined that the need for good thermal contact to the TES and fast thermal equilibration of the absorber (to avoid variation of pulse shape with position in the absorber) conflicts with the requirement that the absorber not alter the superconducting transition of the sensor. ${ }^{2}$

We have developed absorbers that are cantilevered over the sensitive regions of the TES's themselves as well as the surrounding area, making contact to the TES only at normal metal features that are already part of its design. We deposit $\mathrm{Au}$ along the edges parallel to the current flow, to ensure a reproducible boundary condition, and on the surface, in interdigitated stripes perpendicular to the current flow, to reduce detector noise ${ }^{3}$. Limiting the absorber contacts to these normal regions avoids alter- ing the properties of the TES thermometer, even when a normal metal is used as the absorber.

In order to avoid shunting the TES bias current through the absorber, the absorber and TES may make contact at only one region along the direction of current flow. This constraint limits the size of the contact area within the TES. To ensure mechanical stability of the cantilevered structure, we extend the contact region onto the membrane. In this letter we present results from electroplated $\mathrm{Au}$ absorbers of two basic designs, one limiting the absorber-TES contact to the device edges and one making contact to the interdigitated stripes, as shown in Figure 1a. A photograph of a portion of an $8 \times 8$ array containing such pixels is shown in Figure 1b.
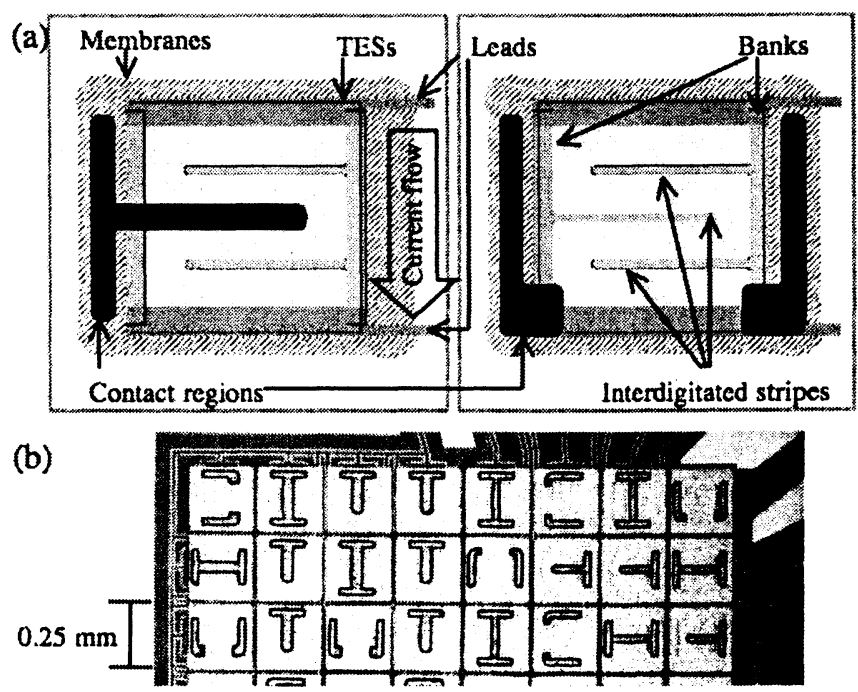

FIG. 1: (a) Schematics of the cantilevered absorbers. The square gray outline marks the edge of the absorbers, which are supported only at the contact areas shown shaded in black. (b) Photograph of TES array with electroplated Au absorbers. 
The area where the absorber touches the membrane potentially provides a path to the heat sink that does not go through the TES. In order to achieve high spectral resolution in such a design, the equilibration time $t_{\mathrm{eq}}$ in the absorber must be much shorter than the time for energy loss $t_{\text {loss. }}$. The ratio $t_{\text {eq }} / t_{\text {loss }}$ needs to be of order $\Delta E / E$. Applying the Wiedemann-Franz law to the lowtemperature electrical conductivity of our $\mathrm{Au}$ absorbers, we estimate that $t_{\mathrm{eq}}$ is approximately $30 \mathrm{~ns}$ at $0.09 \mathrm{~K}$. We further estimate that $t_{\text {loss }}$, the time for energy deposited in one of the on-membrane supports to be lost to the heat sink, is of order $1 \mathrm{~ms}$, based on the heat capacity of the contact region and the conductance from it. Therefore, we expect no significant energy loss from the on-membrane support in either geometry. Also, we expect no significant variation of pulse shape, thanks to the fast $t_{\mathrm{eq}}$.
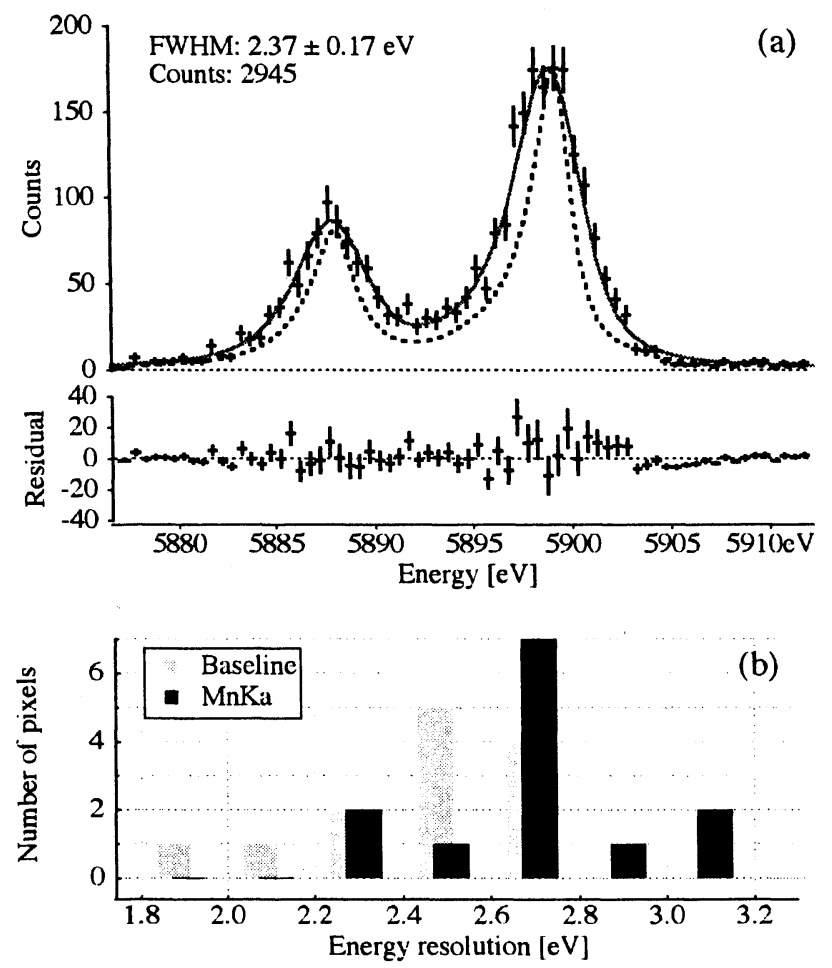

FIG. 2: (a) Optimally filtered pulse height spectrum with Mn $K_{\alpha}$ lines. The solid line shows the least-squares best fit, and the dotted line shows the natural line shape, from Hölzer et al. ${ }^{4}$ with correction and extension provided by Hölzer via private communication. (b) Distribution of $\Delta E_{\mathrm{b}}$ and $\Delta E_{\mathrm{Mn}}$ (FWHM) of 13 devices. The bin size of $0.2 \mathrm{eV}$ is comparable to typical statistical errors associated with the individual spectral fits.

We have fabricated two wafers of TES arrays with electroplated $\mathrm{Au}$ absorbers with a fill factor of $92 \%$. They had 5.3 and $3.7 \mu$ m-thick absorbers, with QE at $6 \mathrm{keV}$ of $99 \%$ and $95 \%$, respectively. In the following discussion, we present the performance of one pixel from the first wafer $\left(T_{\mathrm{c}} \sim 77 \mathrm{mK}\right)$ and 12 pixels from the second wafer
$\left(T_{\mathrm{c}} \sim 88 \mathrm{mK}\right)$. These pixels represent both absorber contact geometries, and the results are sufficiently similar to warrant discussion of the ensemble without specifying type. On one of the devices from the second wafer, we obtained a resolution of $2.37 \pm 0.17 \mathrm{eV} \mathrm{FWHM}$ at 5.9 $\mathrm{keV}$ in a spectrum of $\mathrm{Mn} K_{\alpha}$ lines with $\sim 2900$ counts as shown in Figure 2a. The FWHM was determined from a least-squares fit of the convolution of a Gaussian of that width with the known profile of the Mn $K_{\alpha}$ lines $^{4}$. The quoted error is one standard deviation, assuming statistical errors only. The baseline energy resolution $\Delta E_{\mathrm{b}}$ was $2.33 \pm 0.03 \mathrm{eV}$ FWHM, where $\Delta E_{\mathrm{b}}$ is determined from measurement of the noise. Therefore, there was no significant degradation of the resolution due to energy loss via the absorber supports or due to variation of pulse shape. In Figure 2b, we summarize the results of the 13 pixels. The bias points for these measurements ranged from $7 \%$ to $26 \%$ of $R / R_{\mathrm{n}}$, where $R_{\mathrm{n}}$ is the normal state resistance of $\sim 8 \mathrm{~m} \Omega$, and were not rigorously optimized for each device. Their $\Delta E_{\mathrm{b}}$ is distributed around $2.5 \mathrm{eV} \mathrm{FWHM,}$ while $\Delta E_{\mathrm{Mn}}$ is distributed around $2.7 \mathrm{eV}$ FWHM. This difference is unlikely to be fundamental since repeated spectral acquisitions on the same pixel have resulted in differences in $\Delta E_{\mathrm{Mn}}$ on the same scale, likely due to systematic errors in the measurement such as from thermal and electrical gain instability. We have achieved spectral resolution comparable to the state of the art for TES calorimeters $^{6}$, but in a close-packed array that can be scaled to the focal-plane requirements of Constellation$\mathrm{X}$. The response was fairly linear; the ratio of the $\mathrm{Mn} \mathrm{K}_{\beta}$ and $\mathrm{Mn} \mathrm{K}_{\alpha 1}$ pulse heights after optimal filtering ${ }^{5}$ was $1.087 \pm 0.007$, whereas the corresponding ratio in energy is 1.103 .

In order to construct a physical model for these devices, we measured the complex impedance ${ }^{7} Z$ of one of the pixels from the second wafer at twelve bias points from 11 to $91 \%$ of $R / R_{\mathrm{n}}$. The impedance curves of the pixel trace semi-circles in the $\operatorname{Im}[Z]$ vs. $\operatorname{Re}[Z]$ plane between $10 \mathrm{~Hz}$ and $1 \mathrm{kHz}$. Such data are well fit by a simple calorimeter model equivalent to a single heat capacity $C$ connected to the heat sink through $G$. We fit the 12 sets of data simultaneously to determine $\alpha$ and $\beta$ (三 $d \log R / d \log T$ and $d \log R / d \log I$, respectively, where $R$, $T$, and $I$ are the resistance, temperature, and current of the TES) of the individual bias points. We let $C$ vary, constraining its value to be same at all 12 bias points. Although the TES heat capacity may change through the transition, this is a valid approximation since the obtained total heat capacity of $1.44 \mathrm{pJ} \mathrm{K} \mathrm{J}^{-1}$ is more than an order of magnitude higher than the maximum TES heat capacity. We fixed $G$ at $149 \mathrm{pW} \mathrm{K}^{-1}$ as determined from measuring the power required to bias the detector over a range in $T_{\mathrm{b}}$. With this simple model, we are able to reproduce the data well.

We also accumulated noise spectra at the same bias points and compared these to the noise predicted by our model. The parameters determined from fitting the impedance curves reproduce the low-frequency portion of 
the noise spectra, where noise from thermal fluctuations between the calorimeter and the heat sink dominate. At frequencies corresponding to time scales faster than the pulse recovery time constant $\tau$, Johnson noise and excess white noise dominate. For the purpose of this paper, we define Johnson noise as voltage noise with power $4 k_{B} R T$ $\mathrm{V}^{2} / \mathrm{Hz}$, where $k_{B}$ is Boltzmann's constant, and ignore any corrections for non-Ohmic resistance ${ }^{8}$. Thus, we define excess white noise as the quadrature difference between the measured noise and Ohmic Johnson noise. We fit the noise spectra by fixing all the parameters determined from the impedance fits and varying only the ratio of the excess noise to the Johnson noise. In the model, the Johnson and excess noise are applied as voltage noise sources in series with $R$.

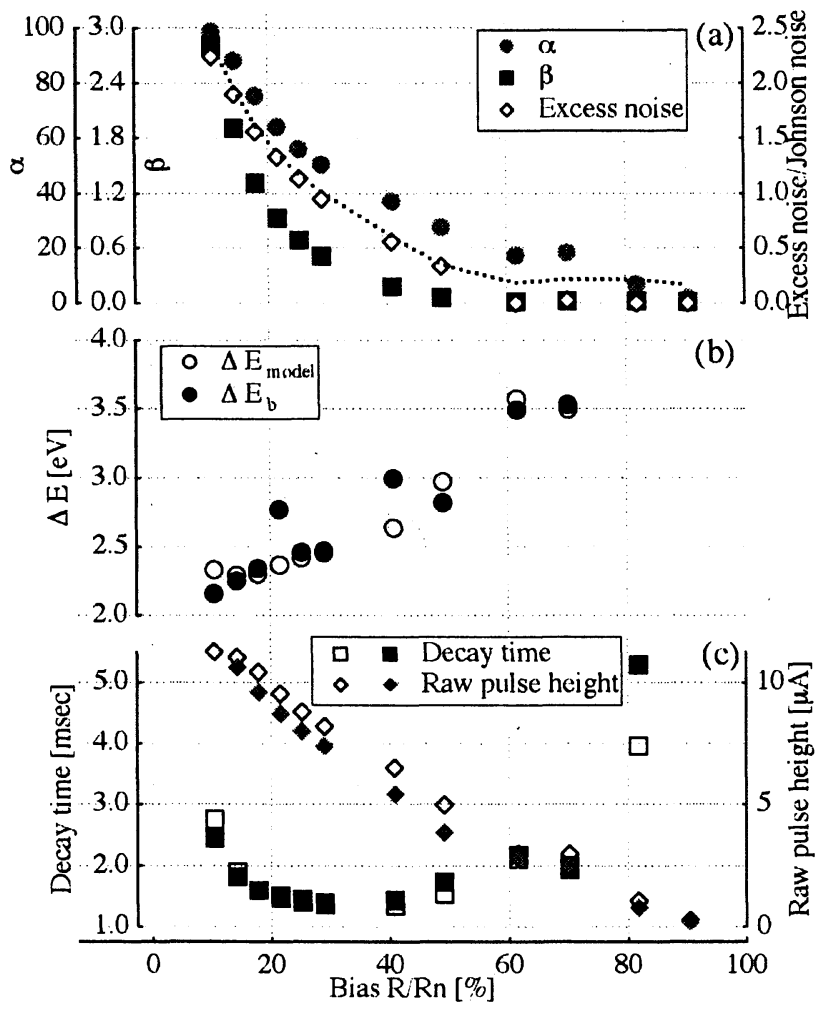

FIG. 3: (a) Circles, squares, and diamonds show $\alpha, \beta$, and excess noise, respectively, vs. bias point. The dotted line, to be read using the excess-noise axis, shows $\sqrt{2 \beta}$ using the $\beta$ determined at each bias point. (b and c) Circles, squares, and diamonds show $\Delta E, \tau$, and raw pulse height, respectively, vs. bias point. Filled markers are actual data and open markers are simulations.

Figure 3a shows the resulting best fit values of $\alpha, \beta$ and excess noise through the transition. The $\alpha$ and $\beta$ values are well correlated. Their relationship is a manifestation of the surface in $R-I-T$ space that describes this particular superconducting transition. The excess noise also follows $\alpha$ and $\beta$. Irwin ${ }^{8}$ estimated that the voltage noise power of a non-Ohmic resistor near equilibrium is $4 k_{B} R T(1+2 \beta) \mathrm{V}^{2} / \mathrm{Hz}$ and our measurement is consistent with that estimate, despite the fact that the devices are operated far from equilibrium.

From the detector parameters determined from our fits, we calculated the theoretical energy resolution $\Delta E_{\text {model }}$ at each point in the transition. As seen in Figure $3 \mathrm{~b}$, the values of $\Delta E_{\text {model }}$ follow $\Delta E_{\mathrm{b}}$ fairly well, although the latter suffers from systematic and statistical errors. (Note that the $\Delta E_{\mathrm{b}}$ values in Figure $2 \mathrm{~b}$ were determined from data records with better statistics.) At the same bias points, we also averaged signal pulses from Mn $K_{\alpha}$ x-rays and compared them to pulses simulated using the parameters determined in the impedance fit. As shown in Figure 3c, the values for $\tau$ of the actual and simulated pulses are in good agreement through the transition. To first order, $\tau$ is proportional to $(C / \alpha) / G$. A non-zero $\beta$ suppresses changes in $R$, which increases $\tau$. Our use of a relatively large bias resistance, $R_{\mathrm{s}}$, of 0.5 $\mathrm{m} \Omega$ weakens electrothermal feedback ${ }^{1}$ in the lower part of the transition, also lengthening $\tau$. These additional effects are reproduced by the simulation. The dependence of the raw pulse heights on the bias point is also explained well by the simulation, as shown in Figure 3c. The dependence of $\Delta E$, raw pulse height, and $\tau$ on bias point was qualitatively similar for the other 12 pixels, but there was a slight systematic difference between the two absorber contact designs.

In order to reduce $\tau$ and $\Delta E_{\mathrm{b}}$, we plan to reduce $C$ by substituting electroplated $\mathrm{Bi}$ for a fraction of the $\mathrm{Au}$, which will also result in decreased linearity and increased $t_{\mathrm{eq}}$ in the absorber. The optimal balance between these effects must be determined. We also plan to reduce $R_{\mathrm{s}}$ and increase $G$ to reduce $\tau$.

In summary, we have achieved high spectral resolution in arrays of close-packed TES x-ray calorimeters in a design scalable for use in astrophysics applications. Moreover, we have characterized these devices and have modeled all aspects of their performance.

The authors are grateful to Kent Irwin's group at NIST Boulder for providing the SQUID amplifiers used for these measurements. They are also grateful to Mark Lindeman at the University of Wisconsin and Tarek Saab at the University of Florida for useful discussions. This work was supported in part by an appointment to the NASA Postdoctoral Program (S.J. Smith and A.-D. Brown) at Goddard Space Flight Center, administered by Oak Ridge Associated Universities through a contract with NASA. 
tinis, App. Phys. Lett. 69, 1945 (1996).

2 C. A. Kilbourne, S. R. Bandler, A. D. Brown, J. A. Chervenak, E. Figueroa-Feliciano, F. M. Finkbeiner, N. Iyomoto, R. L. Kelley, F. S. Porter, T. Saab, et al., in Proc. SPIE (2006), vol. 6266, p. 626621.

3 J. N. Ullom, W. B. Doriese, G. C. Hilton, J. A. Beall, S. Deiker, W. D. Duncan, L. Ferreira, K. D. Irwin, C. D. Reintsema, and L. R. Vale, Appl. Phys. Lett. 84, 4206 (2004).

4 G. Hölzer, M. Fritsch, M. Deutsch, J. Härtwig, and E. Förster, Phys. Rev. A 56, 4554 (1997).
5 S. H. Moseley, R. L. Kelley, R. J. Schoelkopf, A. E. Szymkowiak, and D. McCammon, IEEE Trans. Nucl. Sci. 35, 59 (1988).

6 J. N. Ullom, J. A. Beall, W. B. Doriese, W. D: Duncan, L. Ferreira, G. C. Hilton, K. D. Irwin, C. D. Reintsema, and L. R. Vale, Appl. Phys. Lett. 87, 194103 (2005).

7 M. A. Lindeman, S. R. Bandler, R. P. Brekosky, J. Chervenak, E. Figueroa-Feliciano, F. Finkbeiner, M. J. Li, and C. A. Kilbourne, Rev. Sci. Inst. 75, 1283 (2004).

${ }^{8}$ K. D. Irwin, Nucl. Inst. and Meth. A 559, 718 (2006). 